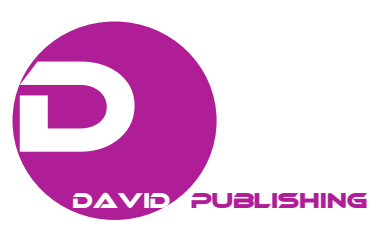

\title{
Cooling of the Vulcain Nozzle's Divergent Wall
}

\author{
Nassir Chellou ${ }^{1}$, Maamar Guendez ${ }^{1}$ and Abdallah Benarous ${ }^{1,2}$ \\ 1. Department of Mechanical Engineering, Faculty of Technology, Hassiba Benbouali University of Chlef, Chlef 02000, Algeria \\ 2. LCEMSM (Laboratory of Control, Tests, Measures and Mechanical Simulation), UHB, Chlef 02000, Algeria
}

\begin{abstract}
Rocket engine nozzle is a propelling nozzle used to expand and accelerate the combustion gases produced by burning propellants to supersonic exit velocities. To furnish high performance and thrust, a maximum of the energy which is released inside the combustion chamber due to the reaction of the propellant and the oxidizer has to be converted into kinetic energy. This converted energy emits high heat fluxes that damage the nozzle that is so expansive to realize. For this matter, lots of researches were established to invent multiple cooling techniques in a way to avoid the damage of the nozzle, so recovering it for to be operative again. This work aims to identify the quality of cooling using the method of film injection. A numerical simulation is run on a scale model of a BKE propulsive nozzle. A parietal injection is conducted in the divergent section where the heat fluxes are enormous what allows us to predict the static temperature \& pressure on the walls without parietal injection \& then we include it in the parameters in order to see the efficiency of the film cooling technique in the nozzles. For this study, we used two different calculation codes: Ansys-Fluent was used to realize the simulation of the supersonic flow \& the parietal injection, where Solidworks simulation was used in the thermal study. The effects of the thermal fluxes $\&$ pressure after $\&$ before the fluidic injection on the wall are discussed.
\end{abstract}

Key words: Propulsive nozzle, fluidic injection, heat fluxes.

\section{Nomenclature}

$\begin{array}{ll}\text { A } & \text { Cross-sectional area } \\ \text { Cp } & \text { Heat capacity at constant pressure } \\ \text { K } & \text { Thermal conductivity } \\ \text { P } & \text { Static pressure } \\ \text { Q } & \text { Rate of heat conduction } \\ \text { V } & \text { Modulus of the velocity vector }\end{array}$

Greek symbols

$\eta \quad$ Cooling efficiency index

$\in \quad$ Section's ratio

Indexes

$\begin{array}{ll}\text { div } & \text { Divergent nozzle } \\ \text { ch } & \text { Combustion chamber } \\ \text { coolant } & \text { Film cooling }\end{array}$

Acronyms

JANAF Joint Army Navy Air Force (organisme)

TOC Thrust Optimized Contour

Corresponding author: Nassir Chellou, student researcher, M.Sc., research fields: thermo fluid control and heat transfer in equipment. E-mail: meca.indu@hotmail.fr.

\section{Introduction}

The Rocket nozzles are designed in way to resist the high pressure \& fluxes, the chemical propulsion using the expanded thermal energy during the combustion of the solid or liquid propellants, remains the only way of the propulsion that is able to provide the thrust exigencies.

The burned gazes are extremely hot, which require a continued cooling of the walls, in condition to not alter the flow's enthalpy [1]. The regenerative cooling techniques that consist on circulating a liquid in a tubing system that surrounds the nozzle, are limited for the motors with medium thrust. When the first stage engines, can only be cooled with a fluidic injection inside the divergent part of the nozzle due to cycle problems.

This technique is not only designed to guarantee a good cooling efficiency, but it contributes also in delaying the restrained lift off $\&$ improves the thrust vectoring [2].

In this work, we interest to study the efficiency of the fluidic injection of the gaseous $\mathrm{H}_{2}$ in the divergent part 
of the subscale nozzle of an optimized contour (TOC) akin to the Volvo-Aero installed in the cryogenic engine Vulcain2 [3]. The temperature \& pressure value are to be obtained from the numerical simulation with Ansys-Fluent program, and will be used directly as boundary conditions in Solidworks in a way to examine the effect of high thermal fluxes \& pressure of the downstream wall of the fluidic injection.

\section{Calculation}

\subsection{Thermodynamic Calculation}

According to the methodology of JANAF [4], the ideal performances relations consider the mixture of the resulting gas of combustion as a perfect gas constant specific heats and relaxing isentropically and dimensional in parts. Considering a diagrammed propulsive chamber with an open volume on one of its faces with the section $A_{c h}$, and/or operates a combustion with $P_{c h}$ pressure, defined as the initial generating pressure (Fig. 1).

The flow characteristics of relaxation can be obtained from the energy \& mass conservation laws associated to the equations of the isentropic. When relating the generating conditions to the exit ones, the energy equation is written as follow:

$$
C p d T+V d V=0
$$

\subsection{Calculation of the Thermal Flux Effect on the} Downstream Wall

The thermal flow characteristics are calculated using the fundamental thermodynamic equations, the heat transfer by conduction obeys Fourier's law which states that the rate of heat conduction $Q_{c}$ is proportional to the heat transfer area (A) and the temperature gradient $(d T / D x)(6)$ or:

$$
\mathrm{Q}_{\mathrm{c}}=-\mathrm{KA}(\mathrm{dT} / \mathrm{dx})
$$

where, $\mathrm{K}$ is the thermal conductivity, measures the ability of a material to conduct heat. The rate of heat conduction is given by Ref. [7]:

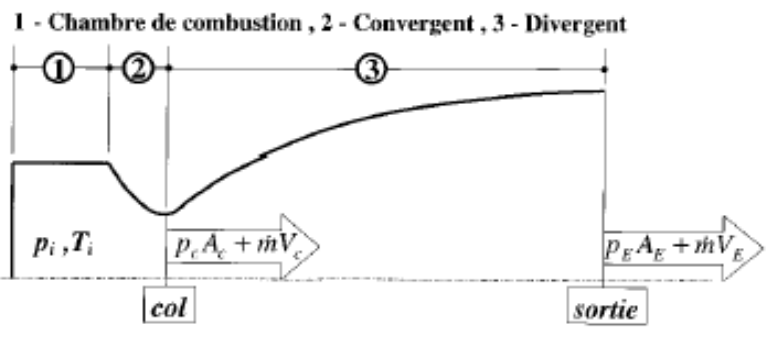

Fig. 1 Schematic of a propulsive chamber.

$$
\mathrm{Q}_{\mathrm{c}}=-\mathrm{KA}\left(\mathrm{T}_{\mathrm{H}}-\mathrm{T}_{\mathrm{C}}\right) / \mathrm{L}
$$

The parameters of temperature are taken from the thermodynamical study with Ansys-Fluent simulation [1].

\section{Model Description \& Operating Conditions}

The nozzle which we carried our simulation on is the one called BKE-DLR [6] represented in 1/8.3 sub model of the Vulcain2 nozzle (Fig. 2). This model is used to sunder the phenomenon inherent cooled nozzles, such as the lift offs and the reinflamation.

The combustion chamber is powered by $\mathrm{H} 2-\mathrm{O} 2$ with a pressure bars and a ration of injection mixture. The section ratios fixed at for the divergent's outlet and at for the convergent's inlet.

The gaseous hydrogen is injected on the section ration. The profile of the divergent (Fig. 3) is numerically constructed with a polynomial defines per pieces [7].

\section{Results and Discussions}

\subsection{Baseline Case (without Injection)}

A non-uniform mesh of 34,040 quadrilateral cells was used for the flow simulation (Fig. 4).

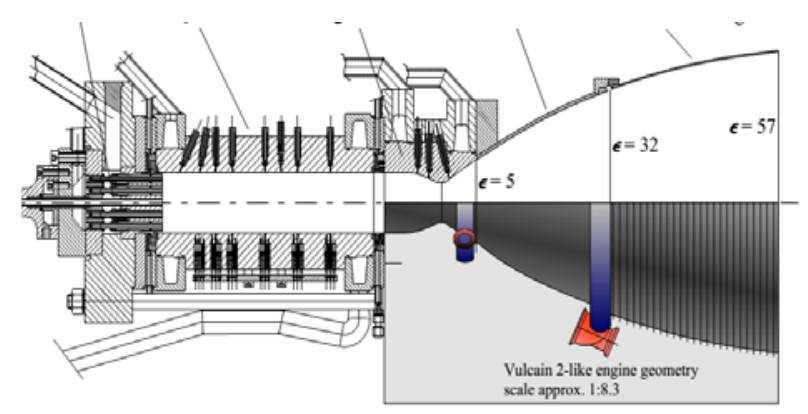

Fig. 2 Side view of the experimental device BKE [4]. 


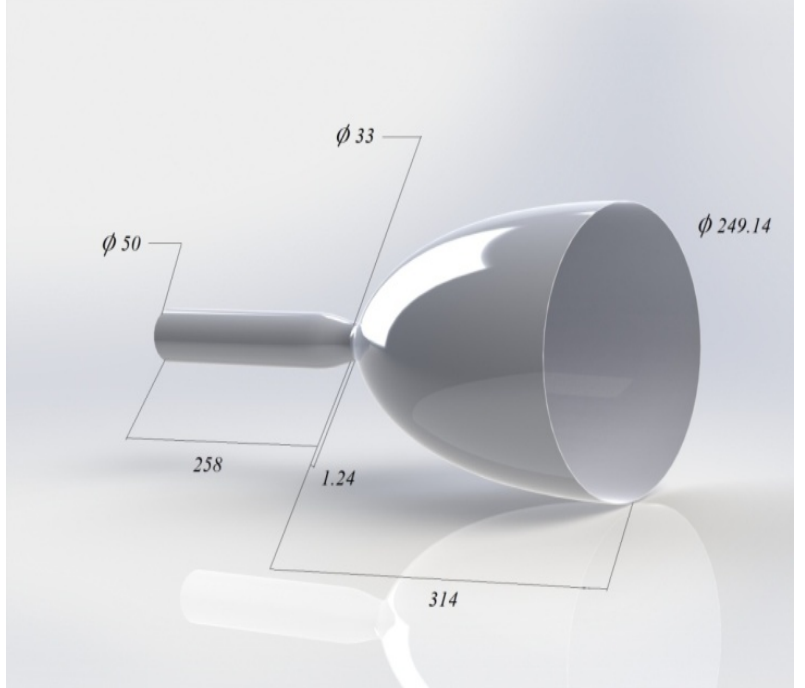

Fig. 3 Geometric model for the BKE nozzle.

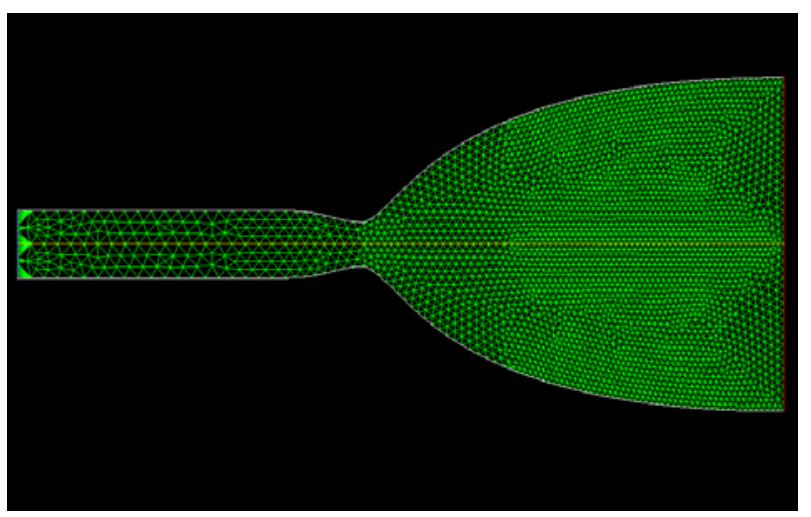

Fig. 4 Geometric mesh for the computational domain.

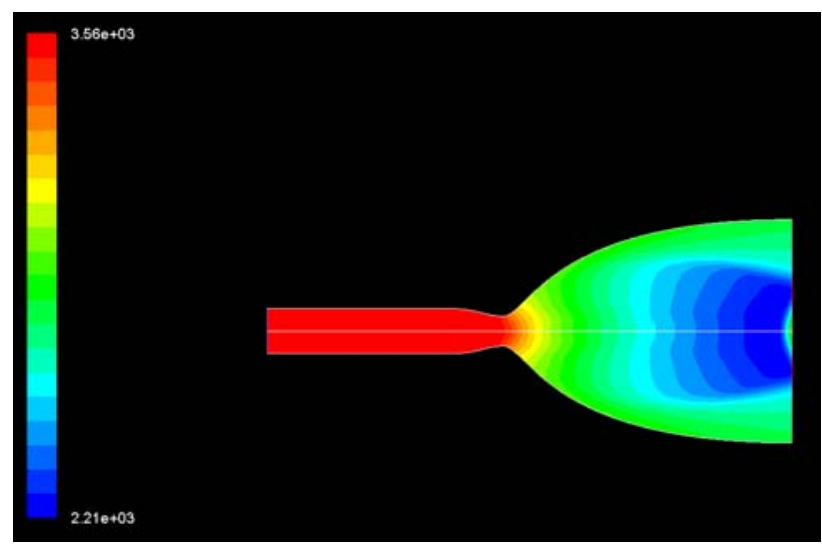

Fig. 5 Spatial contours of temperature (case without fluidic injection).

The simulation was executed in two cases, the first was without the fluidic injection when the second included the injection. The flow simulation helps to exanimate the contours of temperature in the divergent part, where the injection is installed (Fig. 5).
A thermal study based on the flow simulation results, with a uniform triangular mesh of 254,193 cells (Fig. 6).

The simulation is conducted to see the effect of heat fluxes on the walls (Fig. 7) in the interval of $0.11077902696 \mathrm{~m}$, to $0.314 \mathrm{~m}$ (from the injection section, to the outlet one).

The results show that the fluxes are high in comparison to the thermal resistance of the AISI 1018 used in the construction of the nozzle, these thermal deformation cause loses in the physical properties of the material what make it unable for a second use.

\subsection{Case with Fluidic Injection}

In this case the Hydrogen $\left(\mathrm{H}_{2}\right)$ is injected perpendicularly on the divergent wall with a pressure of 0.17 bars and a temperature of $294 \mathrm{~K}$, which represent a fraction of $4 \%$ of the nozzle's flow [1].

The evolution of the static temperature in the downstream section (Fig. 8) shows a remarkable drop

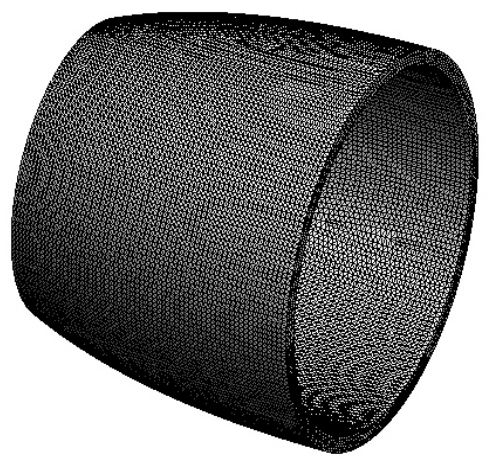

Fig. 6 Geometric mesh (case without injection).

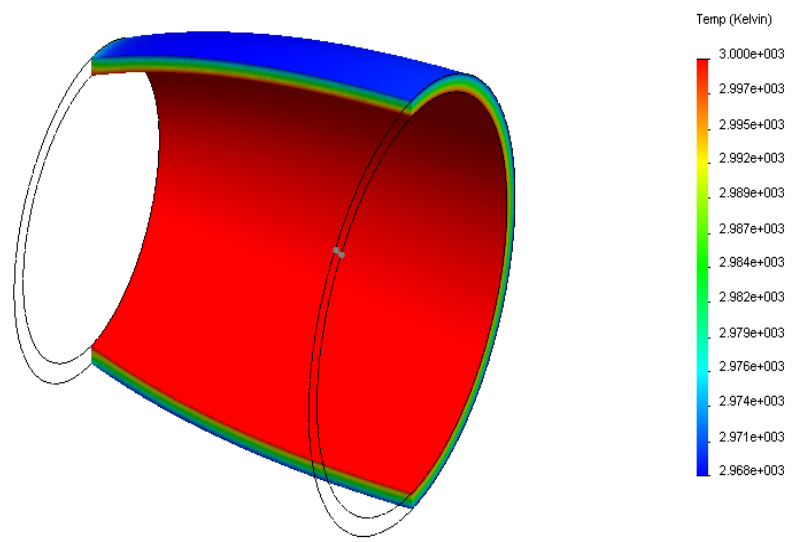

Fig. 7 Thermal deformation (case without injection). 


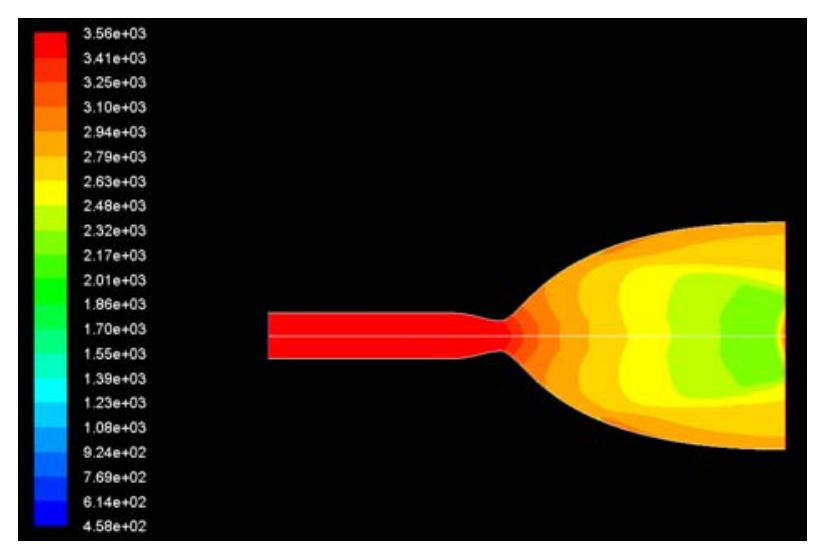

Fig. 8 Spatial contours of temperature (case with fluidic injection).

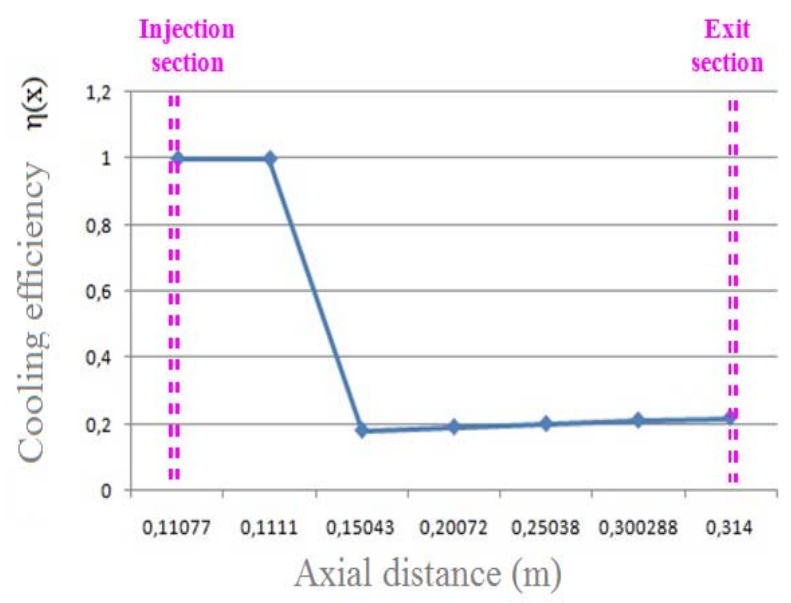

Fig. 9 Cooling efficiency distribution.

in the total temperature in the divergent part, what prove the positivity of the cooling efficiency, that is presented in a dimensionless factor measuring the gap between the wall's temperature $T_{\text {div }}(x)$, the cooling film temperature $T_{\text {Coolant }}$ and the total temperature of the burned gases $T_{c h}$, as following [1]:

$$
\eta_{\text {cooling }}(x)=\frac{T_{c h}-T_{\text {div }}(x)}{T_{c h}-T_{\text {coolant }}}
$$

Fig. 9 shows the cooling efficiency in the downstream extension of the injection zone.

The distribution on the figure shows that over $35 \%$ (around $110 \mathrm{~mm}$ on an axial distance) of the divergent part keeps a unit value, mean equality between the wall $\&$ the cooling fluid. The wall's temperature gets affected by the burned gases one inducing a $60 \%$ drop in the cooling efficiency around $150 \mathrm{~mm}$. The injection's boundary conditions guarantee a good cooling for the last part of the divergent where we assist some cooling efficiencies of $20 \%$ order [1].

In order to see the cooling efficiency on the wall, a thermal study is conducted starting from the injection part to the exit. With a uniform mesh of 335,733 cells with a global contact feature between the wall and the injected fluid (Fig. 10), the simulation reports a thermal deformation contour (Fig. 11) that shows a remarkable efficiency in the cooling of the walls with a drop of around $1,800^{\circ} \mathrm{K}$.

\section{Comparison of the Simulation's Results}

In order to see the effects of our research, a comparison should be done in order to make the results more valuable, where multiple probes are allocated along the geometry's body (axial cut plot) in order to see the distribution of the temperature there, the results are shown in Fig. 12.

The temperature in the first case seems stable where

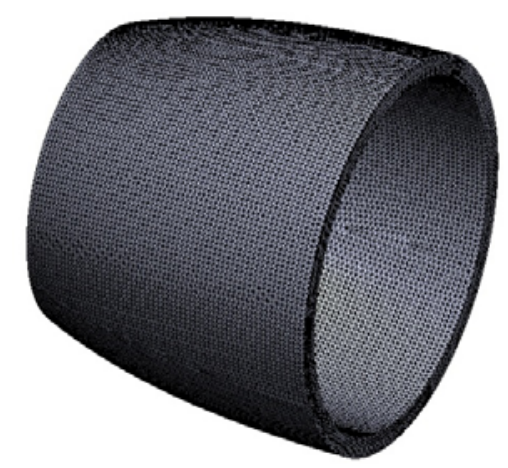

Fig. 10 Geometric mesh (case with injection).

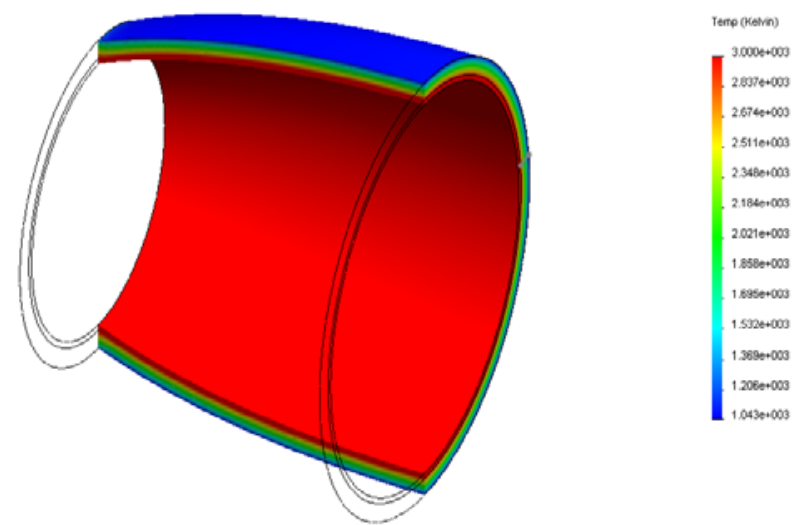

Fig. 11 Thermal deformation (case with injection). 


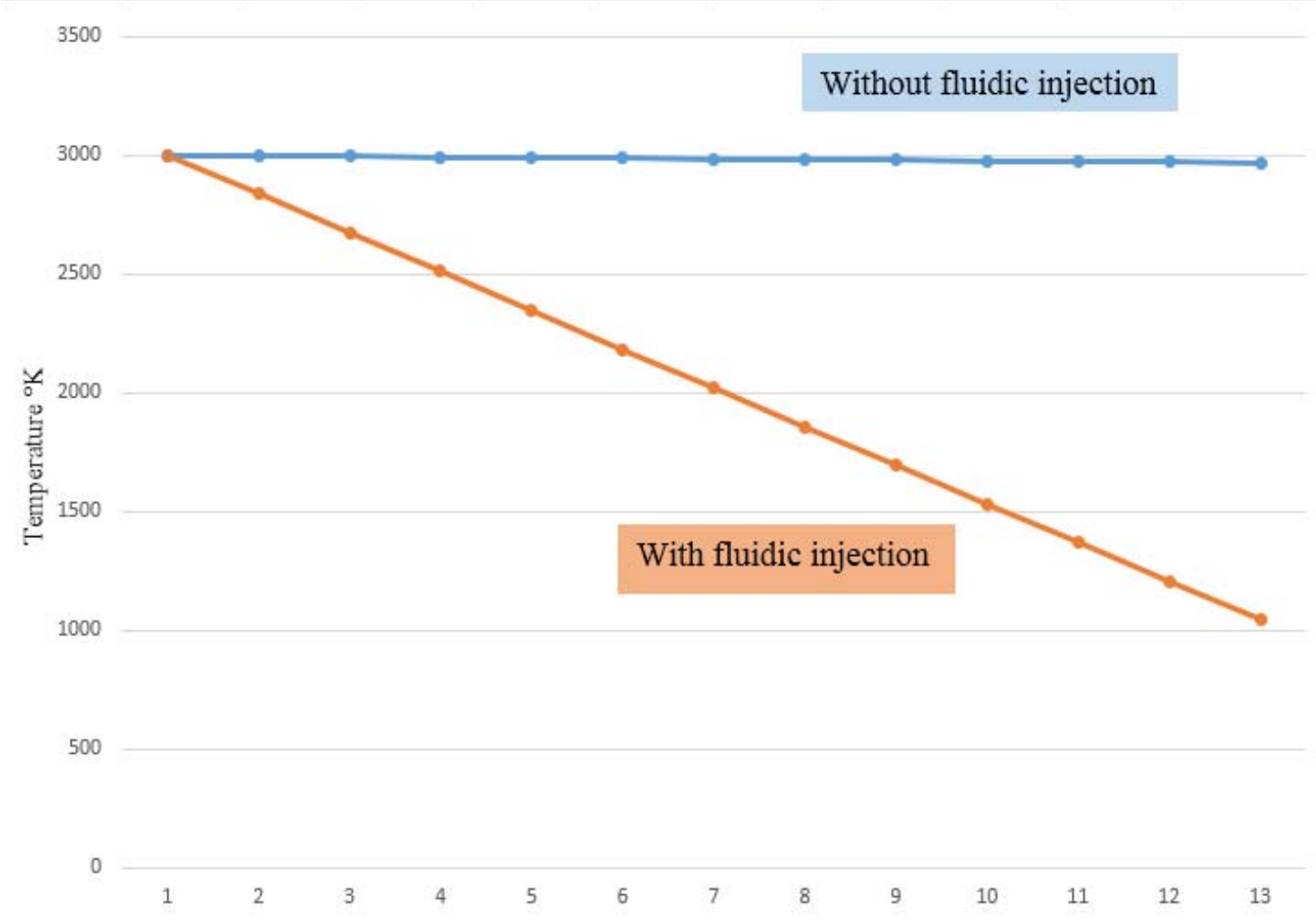

Fig. 12 Distribution of the temperature along the nozzle's body (axial cut plot).

it is slightly approaching the max value of $T=3,000^{\circ} \mathrm{K}$, however, while fluidic injection a remarkable drop appears clearly, which helps to phrase the bellow conclusion.

\section{Conclusions}

A numerical simulation was conducted on the ambient temperature in the subscale model of the BKE nozzle subjected to a fluidic injection, in order to see the efficiency of cooling with the technique of film cooling.

The conducted simulation indicates that the thermal resistance of the AISI 1018 steel shows a fatigue in front of the high temperature loads on the walls (even its high toughness, strength and ductility performances), which requires cooling to resist until the thrust.

We replied to this need with a perpendicular fluid injection $\left(\mathrm{H}_{2}\right)$ on the divergent wall. The thermal characteristics of the injected fluid ( $T=26 \mathrm{k}$, thermal conductivity $\left.=0.182 \mathrm{~W} / \mathrm{m}^{\circ} \mathrm{C}[8]\right)$ results a remarkable drop of the temperature $(\approx 45 \%)$ where the efficiency of the injected fluid appears.

Counter to other cooling techniques of the nozzle, it seems that the fluid injection is the most efficient method, looking that we avoid lots of common problems, as the hydraulic losses and cycle problems (liquid phase) in the regenerative cooling, or even the short firing time in the ablative cooling.

Thus, we insist to say that the film cooling method is still under the test bench, not yet realized, due to the required precision to avoid the boundary layer separation.

\section{References}

[1] Guendez, M., Chellou, N., and Benarous, A. 2014. "Flow Analysis in a Propulsive Nozzle Subjected to a Fluidic Injection." Presented at CIEP'14, Constantine.

[2] Zmijanovic, V., Lago, V., Sellam, M., and Chpoun, A. 2014. "Thrust Shock Vector Control of an Axisymmetric Conical Supersonic Nozzle via Secondary Transverse Gas 
Injection.” Shock Wave 24 (1): 97-111.

[3] Haggander, J. 2001. An efficient Concept-Design Process for the Vulcain2 Thruster, Rapport Technique Volvo-Aero No. 102: Trollhatan, Sweden.

[4] JANAF. 1975. Rocket engine performance prediction and evaluation, CPIA Publication No. 246.

[5] DASSAULT SYSTEMS, Solidworks help, Simulation background.

[6] SOLIDWORKS customer software support.

[7] Guendez, M. 2014. "Analysis of the Film Cooling for a Propulsive Nozzle." Master II Project in Mechanical Engineering, University of Hassiba Benbouali, Chlef, Algeria.

[8] http://www.engineeringtoolbox.com/. 